\title{
Wireless HART stack using multiprocessor technique with laxity algorithm
}

\author{
A. Manjunathan ${ }^{1}$, E. D. Kanmani Ruby², W. Edwin Santhkumar ${ }^{3}$, A. Vanathi ${ }^{4}$, P. Jenopaul, \\ S. Kannadhasan ${ }^{6}$ \\ ${ }^{1}$ Department of Electronics and Communication Engineering, K. Ramakrishnan College of Technology, Samayapuram, \\ Trichy, India \\ ${ }^{2}$ Department of Electronics and Communication Engineering, Vel Tech Rangarajan Dr. Sagunthala R \& D Institute of \\ Science and Technology, Chennai, Tamilnadu, India \\ ${ }^{3}$ Department of Mechanical Engineering, Sri Shakthi Institute of Engineering and Technology, Chennai, Tamilnadu, \\ India \\ ${ }^{4}$ Department of Electronics and Communication Engineering, Rajalakshmi Institute of Technology, Chennai, Tamilnadu, \\ India \\ ${ }^{5}$ Department of Electrical and Electronics Engineering, Adi Shankara Institute of Engineering and Technology, Kalady, \\ Kerala, India \\ ${ }^{6}$ Department of Electronics and Communication Engineering, Cheran College of Engineering, Anna University,
} Tamilnadu, India

\begin{tabular}{l} 
Article Info \\
\hline Article history: \\
Received Aug 13, 2021 \\
Revised Oct 21, 2021 \\
Accepted Nov 2, 2021 \\
\hline
\end{tabular}

\section{Keywords:}

GPS

GSM

Index terms-RTOS

Laxity time

Microcontroller

Wireless HART

\begin{abstract}
The use of a real-time operating system is required for the demarcation of industrial wireless sensor network (IWSN) stacks (RTOS). In the industrial world, a vast number of sensors are utilised to gather various types of data. The data gathered by the sensors cannot be prioritised ahead of time. Because all of the information is equally essential. As a result, a protocol stack is employed to guarantee that data is acquired and processed fairly. In IWSN, the protocol stack is implemented using RTOS. The data collected from IWSN sensor nodes is processed using non-preemptive scheduling and the protocol stack, and then sent in parallel to the IWSN's central controller. The real-time operating system (RTOS) is a process that occurs between hardware and software. Packets must be sent at a certain time. It's possible that some packets may collide during transmission. We're going to undertake this project to get around this collision. As a prototype, this project is divided into two parts. The first uses RTOS and the LPC2148 as a master node, while the second serves as a standard data collection node to which sensors are attached. Any controller may be used in the second part, depending on the situation. Wireless HART allows two nodes to communicate with each other.
\end{abstract}

This is an open access article under the CC BY-SA license.

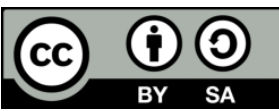

\section{Corresponding Author:}

A. Manjunathan

Department of Electronics and Communication Enigneering

K. Ramakrishnan College of Technology, Samayapuram, Trichy, India

Email: manjunathankrct@gmail.com

\section{INTRODUCTION}

The viability of the suggested architecture in actual product design has been shown by an implemented wireless HART stack. Future problems are also addressed, as well as recommendations for standard improvement. This is necessary to minimise the possibility of collision (and therefore improve communication reliability) and to satisfy the key criterion of industrial application temporal determinism. To do this, all nodes must be perfectly synced, i.e., the synchronisation jitter must be considerably lower than the 
length of time slot [1]-[5]. In addition, the stack designer must ensure that the node is capable of completing all tasks within the specified time frame. The design of protocol stacks has grown more difficult as a result of such time requirements. To begin with, it is difficult for a CPU with limited resources (clock frequency, memory, energy, and supply) to do complex operations in such a short timeframe [6]-[10]. Second, IWSN stacks are often just a portion of the timing-critical activities that the at the same time, the rapidly growing complexity and other special needs of industrial systems have necessitated the use of real-time operating systems (RTOS) in IWSN stacks. However, the introduction of RTOS and multiprocessor support has made ensuring timing integrity more difficult [11]-[15]. A better architecture is required, yet current research on the subject is inadequate.

This article discusses common process automation needs for a variety of applications aimed towards industrial wireless sensor and actuator networks [16]-[20]. Given these criteria, it's clear that today's commercial WSNs aren't designed for industrial automation, since they're mostly driven by consumer or other application requirements. In addition, we outline the main difficulties that must be overcome in order to fully use WSANs to the degree that the industry anticipates [21]-[25].

\section{RELATED WORKS}

Edmon et al. [1] describe an IWSN-based system. In this part, we've gone over some of the main problems that need to be solved in order for IWSN to be deployed on a wide scale. To be competitive and cost-effective. The IWSN that has been suggested is:

- Human safety, environmental protection, and priority should always take precedence. By definition, process automation functions are safety essential, yet the majority of them are not.

- Most information sent to and received from field devices is normalised to the value of the measured item.

- The importance of availability in industrial large-scale manufacturing cannot be overstated. Even little hiccups in communication may result in a major output halt.

- Latency/retransmission data sent in the field network is only useful for a limited period due to the nature of automation. When data is sent to a specific location, it is of limited value in most real-time systems.

Song et al. [2] propose a wireless HART that relies on a central network management for routing and communication scheduling. Physical layer, data connection layer, network layer, transport layer, and application layer are all part of the wireless HART protocol stack, which follows the OSI layer communication paradigm. Akerberg et al. [3] proposed a method that classifies current packet or task scheduling systems based on various criteria. Dead line: packet scheduling methods may be categorised depending on the data packet station's deadline arrival. Priority is given to the extremely essential job when the first work must be completed by the deadline. Real-time scheduling: among all data packets in the queue, real-time data packets get the greatest priority.

In 2011, Hwang et al. [4] when a system processor is available in a multiprocessor environment, ready-to-execute jobs are placed in a priority queue, with the job at the top of the list running the processor. It is based on a laxity time algorithm that prioritises the slack time rate with the least amount of slack time. This is how a scheduler determines how long it will take to execute a job. In 2020, Bhuvaneshwari and Manjunathan [15] the technique for determining which work will be finished and when it will be completed at any given time.

\section{WIRELESS HART}

Wireless HART is a technique for wireless sensor networks. HART-addressable remote transducer (highway addressable remote transducer). It is a multi-vendor, interoperable wireless standard, and wireless HT technical definition that has been created. Wireless HART has a frequency range of $2.4 \mathrm{GHz}$ and is an IEEE802.14 compliant wireless transceiver. It has a programmable $16 \mathrm{~KB}$ flash memory and $4 \mathrm{~KB}$ RAM memory, as well as several 16 bit timers and a USB connection for connecting to a computer. A central network manager is used to provide routing and communication scheduling in wireless HART.

Physical layer, data connection layer, network layer, transport layer, and application layer are all part of the wireless HART protocol stack, which follows the OSI layer communication paradigm. For an IEEE802.15.4, the physical layer is most often utilised in the OSI model. Up to $2.4 \mathrm{GHz}$ in the frequency band. It comes in a direct sequence spread spectrum variant. The channel numbers range from 11 to 26 , with a 5 MHZ gap between neighbouring channels. Layer of datalinks: The time synchronised Data connection layer is a unique characteristic of wireless HART. Wireless HART uses TDMA technology to enable collision-free and predictable communication by defining a tight $10 \mathrm{~ms}$ time window. A transaction in a time slot is described by a vector in wireless HART: [Frame id, index, type, srcaddr, dstaddr, and channelOffset], to name a few. Layers of the network and transport: End-to-end communication for network devices must be 
secure and reliable. (1) field devices, which are attached to the plant process, (2) handheld, which is a portable wireless HART-enabled computer used to configure devices, run diagnostics, and perform calibrations, (3) gateway, which connects host applications with field devices, and (4) a network manager, who is in charge of configuring the network, scheduling, and managing communication between wireless HART devices. Layer of application: responses to various device instructions, data kinds, and status reporting commands and responses are used to communicate between the devices and the gateway.

The whole stack is divided into two processors, as illustrated in Figure 1. Radio processor (processor-I): Only the WHART stack's lowest levels are implemented here. The physical layer, data link layer, and serialization layer are the three layers. The physical and data connection layers are the time-critical layers in this case. A slot time, which is synchronised with the network time source, triggers the actions of sending and receiving a packet. The RTOS sends a signal to the thread when the slot timer interrupts the CPU.

\section{WIRELESS HART}

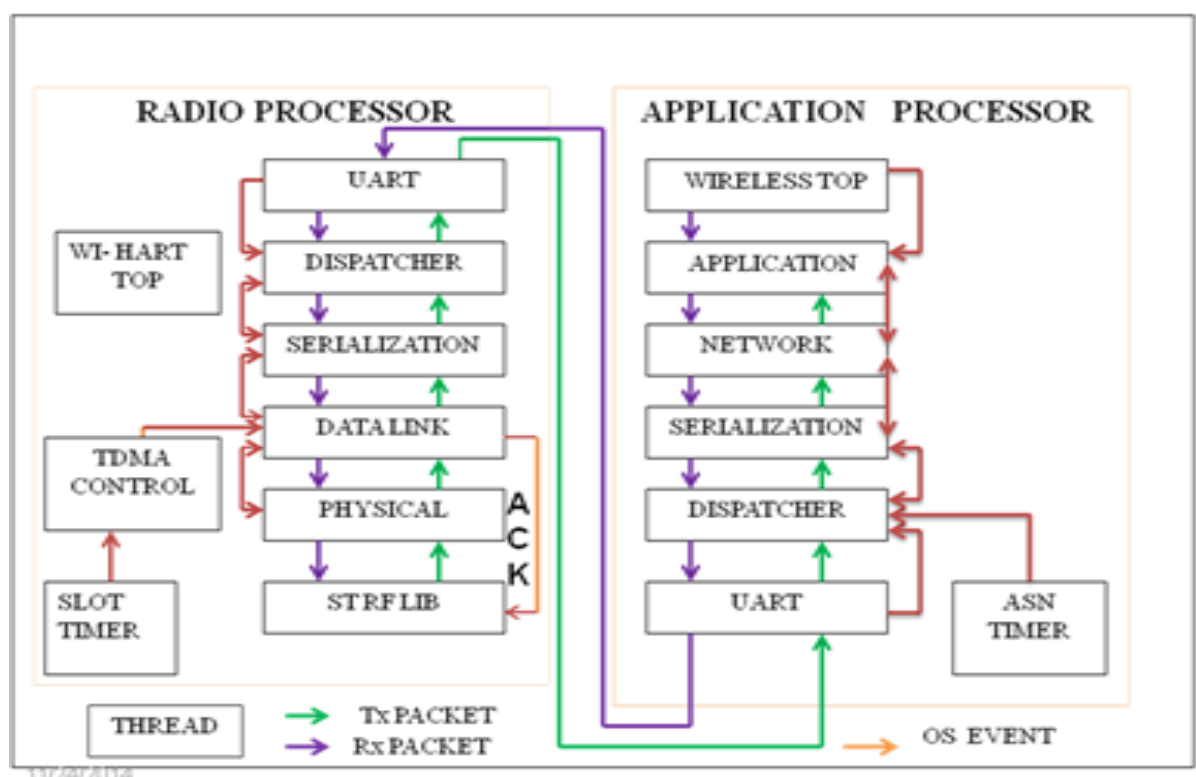

Figure 1. Wireless hart stack

The thread examines the controller to see whether it has received any packets. When a packet is received, the physical layer transfers it to the data link layer's mailbox. The message integrity code is checked by the data link layer (MAC). If the MIC is valid, it generates an ACK packet, encrypts it using the cypher method, and transmits it to the next processor. Hardware acceleration is used to preserve timing integrity. The phrase "optimal scheduling" refers to scheduling techniques that allow all activities to be completed within a predetermined time frame. We present a novel and simple scheduling method in this article.

A hard real-time system must finish all tasks assigned to it by each specified deadline; otherwise, part of the outputs will be worthless, and a major catastrophe may ensue. It is calculated using the least slack algorithm, which is based on non-preemptive scheduling. Di-absolute deadline, t-current time, ri-release time, and di-relative deadline are the least slack rates. Release time, relative deadline, absolute deadline, and execution time are all timing restrictions in real-time systems, as illustrated in Figure 1.The release time (ri) is the time when a task is placed in the ready queue for execution; the relative deadline (Di or di-ri) is the maximum amount of time in which a task should be completed; the absolute deadline (di, or Di+ri) is the time in which a task's execution should be completed; and finally, the execution time (ei) is the time in which a task should be completed.

The LST algorithm works on the principle that the shorter the slack time, the greater the priority. The slack time (di-eir-t) is represented in Figure 2 as the remaining free time (di-eir-t) at the current moment. As illustrated in Figure 3, the eir indicates the time needed to finish the remaining work of a job. All jobs may be scheduled using the LST algorithm if certain conditions are met (1). It may also be regarded as the best algorithm for a single processor. 


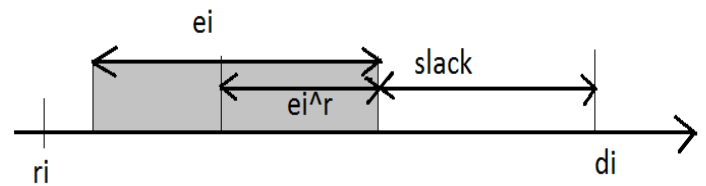

Figure 2. Least slack time

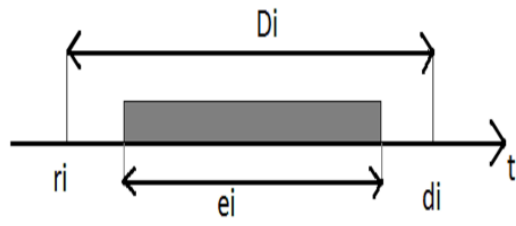

Figure 3. Basic scheduling technique

\section{PROPOSED SYSTEM}

Here data is collected by 3 sensors to an arm processor at the sending end and it is relay to the controller, which is also the arm processor at the receiving end. Here the scheduling for data packet is performed using RTOS. Then here its preemptive scheduling, which resolves of meeting deadlines. Wireless HART segmentation of data received. RTOS is used schedule data packets using non preemptive algorithm to prevent data mismatch and packet collision. The data received is simulated plotted as a graph in GLCD. For existing system RTOS is not used. When data send simultaneously to the central controller is shown in Figure 4. There is chance for data packet collision and data mismatch at receiver.

- Temperature sensor: the LM35 family of temperature sensors are precision integrated-circuit temperature sensors with a directly proportional output voltage to the Celsius temperature.

- When selecting a pressure transducer for a certain application, the first question that typically arises, the kind of device to select absolute gauge or sealed gauge is closely linked to the pressure requirements. Level sensors measure the level of flowing fluids such as liquids, slurries, granular solids, and powders. Liquid level sensors and switches from Gems offer high-reliability monitoring and detection of a variety of fluid media.

- ARM microcontroller: ARM7 controller is the brain of the system. It is a RISC processor and hence can execute code quickly and efficiently. It has several peripherals like a $32 \mathrm{kB}$ to $512 \mathrm{kB}$ flash, $8 \mathrm{kB}$ to 40 $\mathrm{Kb}$ on-chip SRAM. The ARM7 controller has a three stage pipeline, unified bus architecture, a forward compatible code with 32 bit ARM ISA and 16 bit Thumb extension. It has real time trace with ETM9 macro cell. The controller is initially placed in sleep mode. When it is woken up, it finds the source and begins to execute the corresponding instructions. It can control the over all process of the proposed system. It checks for all the inputs and respond for each action to ensure the security as well as reliability is shown in Figure 5.

- Data acquisition node: collection of sensor nodes value reaches through data acquisition node and then sends to microcontroller.

- Sensor fusion: sensor fusion is a technique in which data from many distinct sensors is "fused" to calculate something that no single sensor could discern. Computing the orientation of a gadget in threedimensional space is an example.

- Relay: relays are electromechanical or electronic switches that open and shut circuits. They open and close circuits in another circuit to regulate one electrical circuit. While a relay is usually open (NO), an open contact is present when the relay is not in use.

- A DC motor is based on the principle that similar magnet poles repel each other and unlike magnet poles attract each other. A current flowing through a wire coil produces an electromagnetic field aligned with the coil's centre.

- Zigbee: zigbeeis a set of high-level communication protocols that are used to build personal area networks using tiny, low-power digital radios. The IEEE 802.15.4 standard is used.

- Graphical liquid crystal display (LCD): a graphical LCD is an electronic visual display technology that is utilised in a variety of gadgets and information output sources, most notably in the display screens of electronic devices. This technique uses precise electrical signals to manipulate small crystals in a confined liquid crystal solution to execute graphical display operations on a two-dimensional screen.

Buzzer: a buzzer is a signalling device that is mechanical, electromechanical, magnetic, and electromagnetic. An oscillating electrical circuit or another audio signal source may power a piezo electric buzzer. When a button is pushed, a click beep or ring may be heard. 
Here the proposed system collected by sensor value are gathered from several sensors and to transferred data acquisition node and then send to microcontroller. Mainly sensor fusion processed by an only one sensor value should be control for an temperature sensor value to an monitor dc motor and then controlled. The transferred value is transmission through zigbee to an industrial system and control system. Here stack design for wireless stack by using RTOS implementation. And then non preemptive scheduling process by provided. Then displays the sensor value are graphical LCD particular interval time monitor the value. To meet the medium amount value are monitor and the buzzer device to be activated.

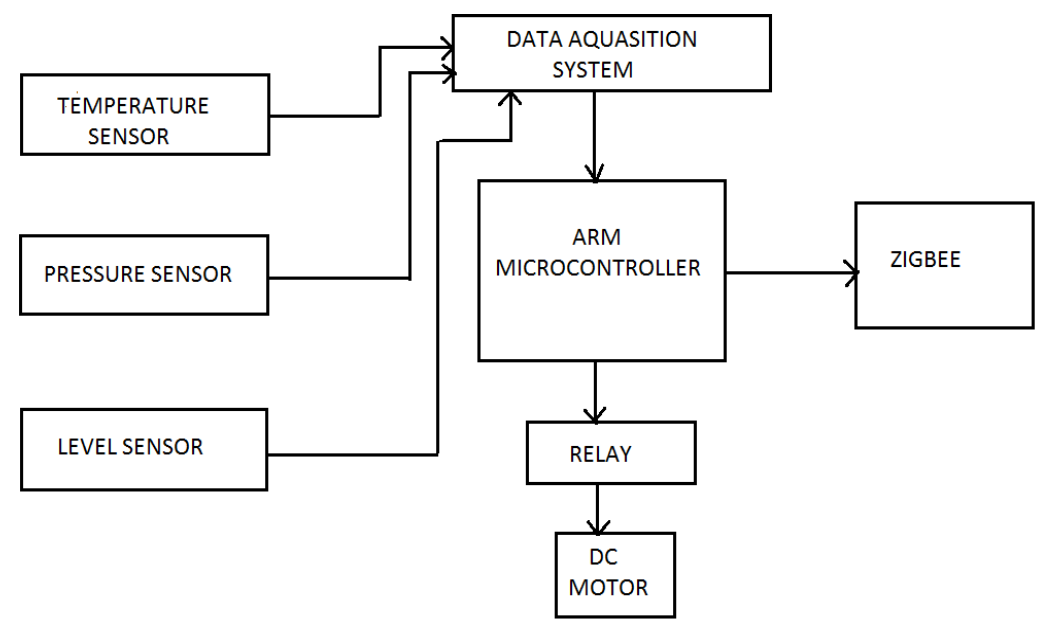

Figure 4. Industrial system

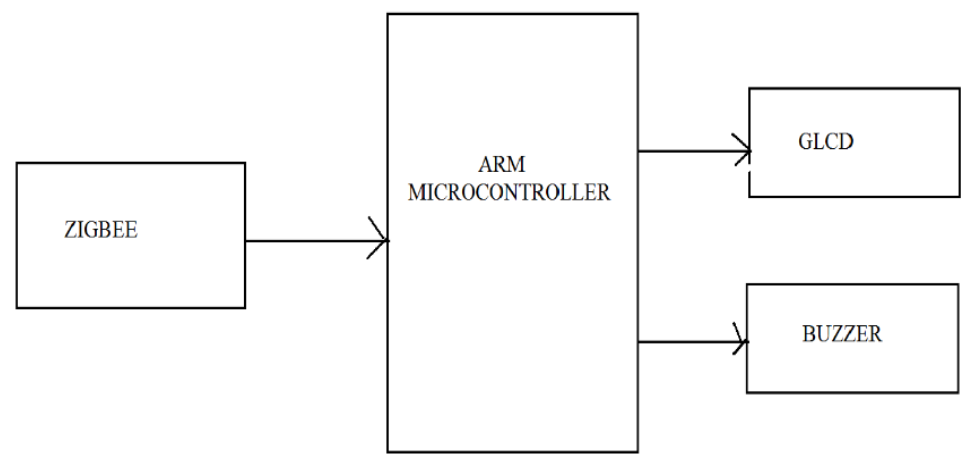

Figure 5. Controller system

\section{CONCLUSION}

WSN find a wide range of applications in every field. Among them the data transfer in the WSN in the industries has lots of issues to be addressed. This project deals with the problems of using a pre-emptive scheduling algorithm in the IWSN. The project employs a non-preemptive scheduling method to transmit data from the sensors to a central controller, which analyses the information received. A new stack has been developed utilising RTOS to accomplish this approach. This stack enables scheduling of the packet transfer in time slots. This part of the project is simulated which has given a successful result.

\section{REFERENCES}

[1] N. Edmonds, D. Stark and J. Davis, "MASS: modular architecture for sensor systems," IPSN 2005. Fourth International Symposium on Information Processing in Sensor Networks, 2005, pp. 393-397, doi: 10.1109/IPSN.2005.1440955.

[2] J. Song et al., "WirelessHART: Applying Wireless Technology in Real-Time Industrial Process Control," IEEE Real-Time and Embedded Technology and Applications Symposium, 2008, pp. 377-386, doi: 10.1109/RTAS.2008.15. 
[3] J. Åkerberg, M. Gidlund and M. Björkman, "Future research challenges in wireless sensor and actuator networks targeting industrial automation," 9th IEEE International Conference on Industrial Informatics, 2011, pp. 410-415, doi: 10.1109/INDIN.2011.6034912.

[4] M. Hwang, D. Choi and P. Kim, "Least Slack Time Rate First: an Efficient Scheduling Algorithm for Pervasive Computing Environment," Institutional Repository, vol. 17, 2011.

[5] M. Hwang, D. Choi and P. Kim, "Least Slack Time Rate First: New Scheduling Algorithm for Multi-Processor Environment," International Conference on Complex, Intelligent and Software Intensive Systems, 2010, pp. 806811, doi: 10.1109/CISIS.2010.20.

[6] A. N. Kim, F. Hekland, S. Petersen and P. Doyle, "When HART goes wireless: Understanding and implementing the WirelessHART standard," IEEE International Conference on Emerging Technologies and Factory Automation, 2008, pp. 899-907, doi: 10.1109/ETFA.2008.4638503.

[7] N. Nasser, L. Karim and T. Taleb, "Dynamic Multilevel Priority Packet Scheduling Scheme for Wireless Sensor Network," IEEE Transactions on Wireless Communications, vol. 12, no. 4, pp. 1448-1459, April 2013, doi: 10.1109/TWC.2013.021213.111410.

[8] S. Zhang, G. Zhang, A. Yan, Z. Xiang and T. Ma, "A highly reliable link scheduling strategy for WirelessHART networks," International Conference on Advanced Technologies for Communications ATC 2013, 2013, pp. 39-43, doi: 10.1109/ATC.2013.6698073.

[9] U. Roedig, S. Rutlidge, J. Brown and A. Scott, "Towards Multiprocessor Sensor Nodes," HotEmNets '10: Proceedings of the 6th Workshop on Hot Topics in Embedded Networked Sensors, 2010, no. 16, pp. 1-5, doi: $10.1145 / 1978642.1978663$.

[10] X. Zhu, S. Han, A. Mok, D. Chen and M. Nixon, "Hardware challenges and their resolution in advancing WirelessHART," 9th IEEE International Conference on Industrial Informatics, 2011, pp. 416-421, doi: 10.1109/INDIN.2011.6034913.

[11] M. D. Udayakumar, G. Anushree, J. Sathyaraj and A. Manjunathan, "The impact of advanced technological developments on solar PV value chain," Materials Today: Proceedings,2021, vol. 45, pp. 2053-2058, doi: 10.1016/j.matpr.2020.09.588.

[12] K. Balachander, G. S. Kumaar, M. Mathankumar, A. Manjunathan and S. Chinnapparaj, "Optimization in design of hybrid electric power network using HOMER," Materials Today: Proceedings, 2021, 45, pp. 1563-1567, doi: 10.1016/j.matpr.2020.08.318.

[13] T. V. Kumar, M. Mathankumar, A. Manjunathan and J. Sathyaraj, "Time based costing of energy storage system with optimal scheduling and dispatch under demand," Materials Today: Proceedings, 2021, vol. 45, pp. 1738-1741, doi: $10.1016 /$ j.matpr.2020.08.620.

[14] C. Bhuvaneshwari and A. Manjunathan, "Reimbursement of sensor nodes and path optimization," Materials Today: Proceedings, 2021, vol. 45, pp. 1547-1551, doi: 10.1016/j.matpr.2020.08.193.

[15] C. Bhuvaneshwari and A. Manjunathan, "Advanced gesture recognition system using long-term recurrent convolution network," Materials Today: Proceedings, vol. 21, pp. 731-733, 2020, doi: 10.1016/j.matpr.2019.06.748

[16] Y. Hu, Y. Ding, L. Ren, K. Hao and H. Han, "An endocrine cooperative particle swarm optimization algorithm for routing recovery problem of wireless sensor networks with multiple mobile sinks," Information Sciences, vol. 300, pp. 100-113, 2015, doi: 10.1016/j.ins.2014.11.052.

[17] S. Yang, U. Adeel, Y. Tahir and J. A. McCann, "Practical Opportunistic Data Collection in Wireless Sensor Networks with Mobile Sinks," IEEE Transactions on Mobile Computing, vol. 16, no. 5, pp. 1420-1433, 1 May 2017, doi: 10.1109/TMC.2016.2595574.

[18] M. Abdelhakim, Y. Liang and T. Li, "Mobile Access Coordinated Wireless Sensor Networks-Design and Analysis," IEEE Transactions on Signal and Information Processing over Networks, vol. 3, no. 1, pp. 172-186, March 2017, doi: 10.1109/TSIPN.2016.2601021.

[19] S. Kannadhasan and R. Nagarajan, "Performance Improvement of Slot Antenna Using Various Parameters and Band Pass Filter," International Conference on Circuits and Systems in Digital Enterprise Technology (ICCSDET), 2018, pp. 1-4, doi: 10.1109/ICCSDET.2018.8821207.

[20] Y. Zhang, S. He and J. Chen, "Near Optimal Data Gathering in Rechargeable Sensor Networks with a Mobile Sink," IEEE Transactions on Mobile Computing, vol. 16, no. 6, pp. 1718-1729, 1 June 2017, doi: 10.1109/TMC.2016.2603152.

[21] S. Singh, P.t Mor and G. Singh, "Application of Embedded Systems in Modern Society," VSRD International Journal of Electrical, Electronics \& Communication Engineering, vol. 2, no. 6, pp. 373-384, 2012.

[22] L. Girod, J. Elson, A. Cerpa, T. Stathopoulos, N. Ramanathan and D. Estrin. Emstar, "a software environment for developing and deploying wireless sensor networks," In Proceedings of the USENIX Conference Proceedings, pages 283-296, 2004.

[23] L. Khelladi, Y. Challal, A. Bouabdallah and N. Badache, "On Security Issues in Embedded Systems: Challenges and Solutions," International Journal of Information and Computer Security, vol. 2, no. 2, 140-174, 2008.

[24] C. J. Chandra, L. Suganya, J. Manjushreekumari, R. Nagarajan and S. Kannadhasan, "Effective Implementation of Efficient Data Collection in WSN," International Research Journal of Engineering and Technology, vol. 8, no. 3, pp. 1311-1316, 2021.

[25] B. Greenstein, D. Estrin, R. Govindan, S. Ratnasamy and S. Shenker. DIFS: A Distributed Index for Features In Sensor Networks. In Proceedings of the IEEE ICC Workshop on Sensor Network Protocols and Applications, Anchorage, AK, April 2003. 